\title{
Endoscopic vacuum therapy with instillation (iEVT) - a novel endoscopic concept for colorectal anastomotic leak and perianal complications
}

\author{
Adam Bobkiewicz, Lukasz Krokowicz, Tomasz Banasiewicz, Maciej Borejsza-Wysocki \\ Department of General, Endocrinological and Gastroenterological Oncology Surgery, Poznan University of Medical Sciences, Poznan, \\ Poland
}

Videosurgery Miniinv 2020; 15 (4): 560-566

DOI: https://doi.org/10.5114/wiitm.2020.93204

\begin{abstract}
Introduction: Anastomotic leaks remain the most fearful complications.

Aim: We present a novel endoscopic salvage therapy known as endoscopic vacuum therapy with instillation (iEVT) as a combination of standard endoscopic vacuum therapy (EVT) and negative pressure wound therapy with instillation (iNPWT).

Material and methods: A case series of 6 consecutive patients treated with iEVT is presented. A Redon drain and a central venous catheter were introduced within polyurethane foam used as a self-made device for iEVT and antimicrobial solution was instilled.

Results: A total of 6 patients with the mean age of $29.5 \pm 8.9$ were treated with IEVT. The mean number of IEVT sessions was $5.8 \pm 2$.3. The mean time of iEVT management was $20.7 \pm 8.8$ days. Locally, a reduction in purulent discharge and defect's dimension with contraction were revealed confirmed with imaging studies.

Conclusions: iEVT is a very encouraging, novel method for complicated perianal diseases and anastomotic leak.
\end{abstract}

Key words: anastomotic leakage, anal fistula, endoscopic vacuum therapy, negative pressure wound therapy.

\section{Introduction}

The introduction of negative pressure wound therapy (NPWT) to clinical practice in the late $90 \mathrm{~s}$ revolutionized the strategy of chronic and complex wounds. In the last decades, NPWT has been implemented in many clinical scenarios and various underlying diseases resulting in higher closure rates when compared to standard wound management [1]. However, there are some important drawbacks of standard NPWT such as bioburden control, painful dressing changes and elimination of thick wound exudates [2]. In 1998 Fleischmann et al. presented the first report of NPWT with instilled antiseptic and an- timicrobial solutions in patients with bone and soft tissue infection [3]. Currently, commercialized devices with negative pressure wound therapy with instillation (iNPWT) are widely used, serving as a strong alternative to standard NPWT. iNPWT combines the standard NPWT with controlled periodic instillation of topical solution to the wound bed. Based on recent comparative studies, iNPWT seems to be positioned as a method of choice in management of chronic and complex wounds when compared to standard NPWT [4].

Although over the past decades significant advances in gastrointestinal surgery have been observed, anastomotic leak remains the most fearful

\section{Address for correspondence}

Adam Bobkiewicz MD, PhD, Department of General, Endocrinological and Gastroenterological Oncology Surgery, Poznan University

of Medical Sciences, 49 Przybyszewskiego St, 60-355 Poznan, Poland, phone: +48 61869 1122, e-mail: bobofon007@gmail.com 
complication after gastrointestinal (GI) surgery. It is associated with a significant rate of morbidity and mortality and affects $5-15 \%$ of patients with a colorectal anastomosis [5]. One of the recently introduced management methods regarding anastomotic complication is endoscopic salvage therapy using stents, fibrin glue, endoscopic clips and endoscopic vacuum therapy. In 2008 Weidenhagen et al. successfully used endoscopic vacuum therapy (EVT) for anastomotic leak after rectal resection [6]. Since then, many reports and case series have been published indicating high success rates of healing anastomotic dehiscence after colorectal anastomosis. EVT was successfully applied in the management of lower GI anastomotic leaks with high success rate ranging from $56 \%$ to $100 \%$ [7].

\section{Aim}

We present a case series of successful application of novel endoscopic salvage therapy using combination of standard EVT and negative pressure wound therapy with instilled antimicrobial solution (iNPWT). The novel technique was termed endoscopic vacuum therapy with instillation (iEVT). Based on our previous experience the application of two well-known techniques simultaneously may increase the success rate of healing anastomotic leaks in patients after colorectal anastomoses as well as in complicated perianal diseases that failed to respond to previous management. In this study we describe a technical instruction on how to prepare an iEVT device and present a case series showing successful application of iEVT in clinical practice.

\section{Material and methods}

The patients were treated between September 2018 and February 2019. A total of 6 patients who underwent iEVT management were analyzed.

\section{Preparation of endoscopic vacuum therapy with instillation (iEVT)}

A thorough and detailed discussion was performed with the patient regarding the method of treatment and the patient signed the written consent for the proposed endoscopic intervention according to our institutional guidelines. Required components for preparing iEVT are presented in Photo 1. Briefly, a piece of polyurethane (PU) foam (Hartmann, Heidenheim an der Brenz, Germany) was trimmed to appropriate dimensions of the fistula cavity measured during the endoscopic evaluation or imaging studies. Next, a tunnel through the long axis of PU foam was made in order to allow for drainage. Preferably, a Redon drain was used because of its stiffness which minimized the potential for collapse of the drain wall when negative pressure was applied. The drain was trimmed at the fenestrated end to adjust the drain's length to the length of PU foam and spanned three fourths of the PU length. The drain was introduced into the PU foam and secured with 2-0 multifilament suture (Ethicon Inc., Somerville, NJ, USA) which was placed at the proximal end of the PU foam and passed through the drain. We routinely did not place another stich at the distal end of the PU foam. The Redon drain was attached to the exudate canister's drain using stoma paste (Stomahesive, Convatec, Poland) and an adhesive dressing to keep the system sealed.

A central venous catheter (CVC) (Two-Lumen CVC, Teleflex, Ireland) was used in order to instill an antimicrobial solution. The tip of the CVC was introduced into the PU foam in the same manner as the Redon drain described above. Such fixation of CVC allows for precise instillation of antimicrobial solution to dissolve and subsequently drain the intra-cavitary exudate (Photo 2). Once the CVC was placed correctly within the PU foam, the CVC was attached to a Redon drain with 2-0 multifilament sutures at many levels to stabilize both drains together (Photo 2).

Antimicrobial solution was delivered using a $20 \mathrm{ml}$ syringe. Two-three times per day an appropriate volume of wound irrigation solution (Prontosan, BBraun,

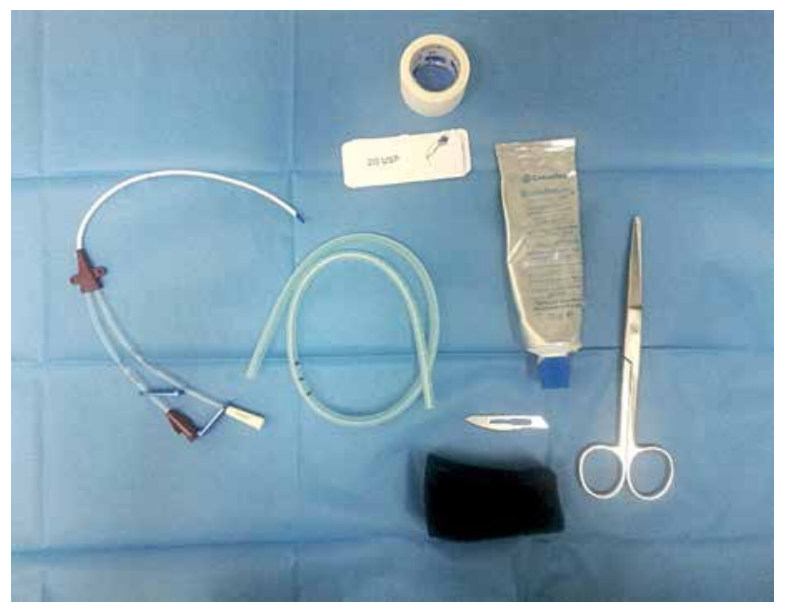

Photo 1. Basic set up for iEVT preparation 


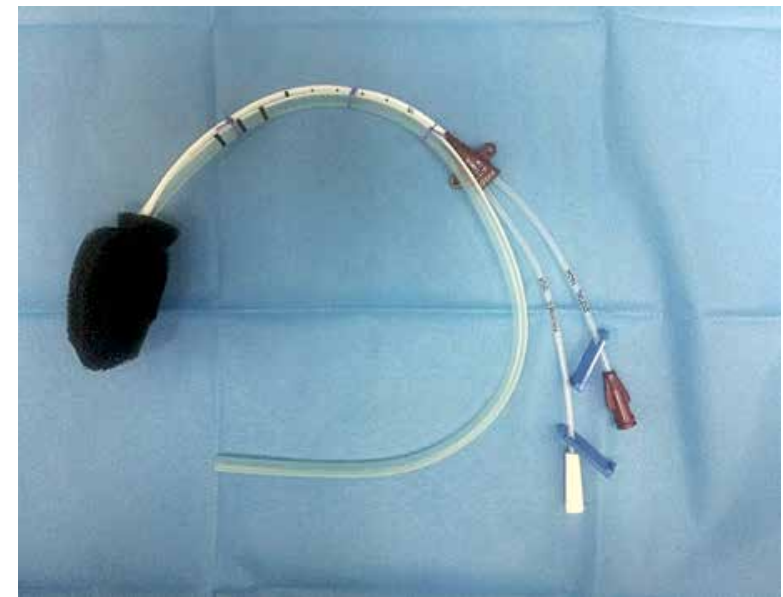

Photo 2. Design of iEVT. Redon drain and central venous catheter (CVC) introduced within polyurethane sponge and secured with sutures

Switzerland or Microdacyn, KikGel, Poland or Ringer solution) was instilled through the CVC's channel. To achieve dwell time (as it is in commercially used iNPWT), 10-minute pause of NPWT treatment was ensured before instillation of antimicrobial solution. Next, negative pressure was applied again to actively drain the instilled antimicrobial solution.

\section{iEVT clinical application}

The procedure was performed in general endotracheal anesthesia and the patient was placed in the lithotomy position. The patient had already received a broad-spectrum antibiotic regimen; therefore perioperative antibiotics were not indicated at this time. PU foam was lubricated with lidocaine gel to facilitate introduction of a device into the $\mathrm{Gl}$ tract. Endosponge of iEVT was introduced thorough the anal canal. Next, under endoscopic guidance, using rat tooth grasper placed through the working channel of the endoscope, iEVT was placed within the fistula cavity. The endoscope was withdrawn with caution to leave the endosponge in place. In complex anal fistula, usually we placed two NPWT systems. First, iEVT was introduced through the anal canal and second placed within the external ostium. Drains were secured to the patient's thigh with tape (Photo 3).

Usually, iEVT was changed every third day. Every next iEVT change, the endosponge was trimmed to smaller dimensions than previous PU foam. The described management allows for a reduction of

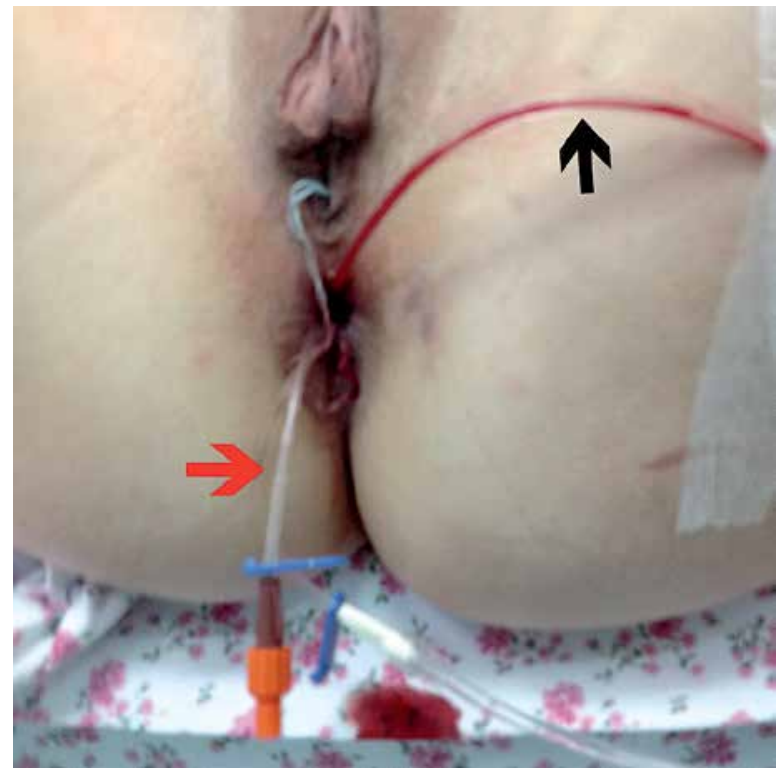

Photo 3. Intraoperative view of iEVT placement. Black arrow indicates EVT drain, red arrow indicates CVC

defect's dimensions and stimulation of the wound bed for gradual proliferation and healing. Every iEVT session, the patient was evaluated endoscopically in terms of the presence of and progressive wound contraction. The patient was also monitored for septic status and progress of her general condition.

\section{Results}

A total of 6 patients were treated with iEVT. There were 3 females and 3 males with the mean age $29.5 \pm 8.9$ years (range: 19-42). Specifications of patients diagnosed with anastomotic leak and perianal diseases treated with iEVT are presented in Table I. The most common underlying pathology was Crohn disease $(n=3)$ and ulcerative colitis $(n=2)$. All patients underwent several previous surgical interventions (Table I). Due to failed previous therapy or complicated course of the primary disease, iEVT was introduced as a method of choice. Before iEVT was initialized, an imaging study (MRI or CT scan) and endoscopy were performed to exclude any concomitant pathology and to establish the strategy of management. A mean session of iEVT per patient was $5.8 \pm 2.3$ (range: $3-9$ ) with the mean total time of the therapy $20.7 \pm 8.8$ days (range: $11-32$ ).

The range of the level of negative pressure (NP) used in iEVT was from -80 to $-140 \mathrm{~mm} \mathrm{Hg}$. The varying level of NP depended individually on the 


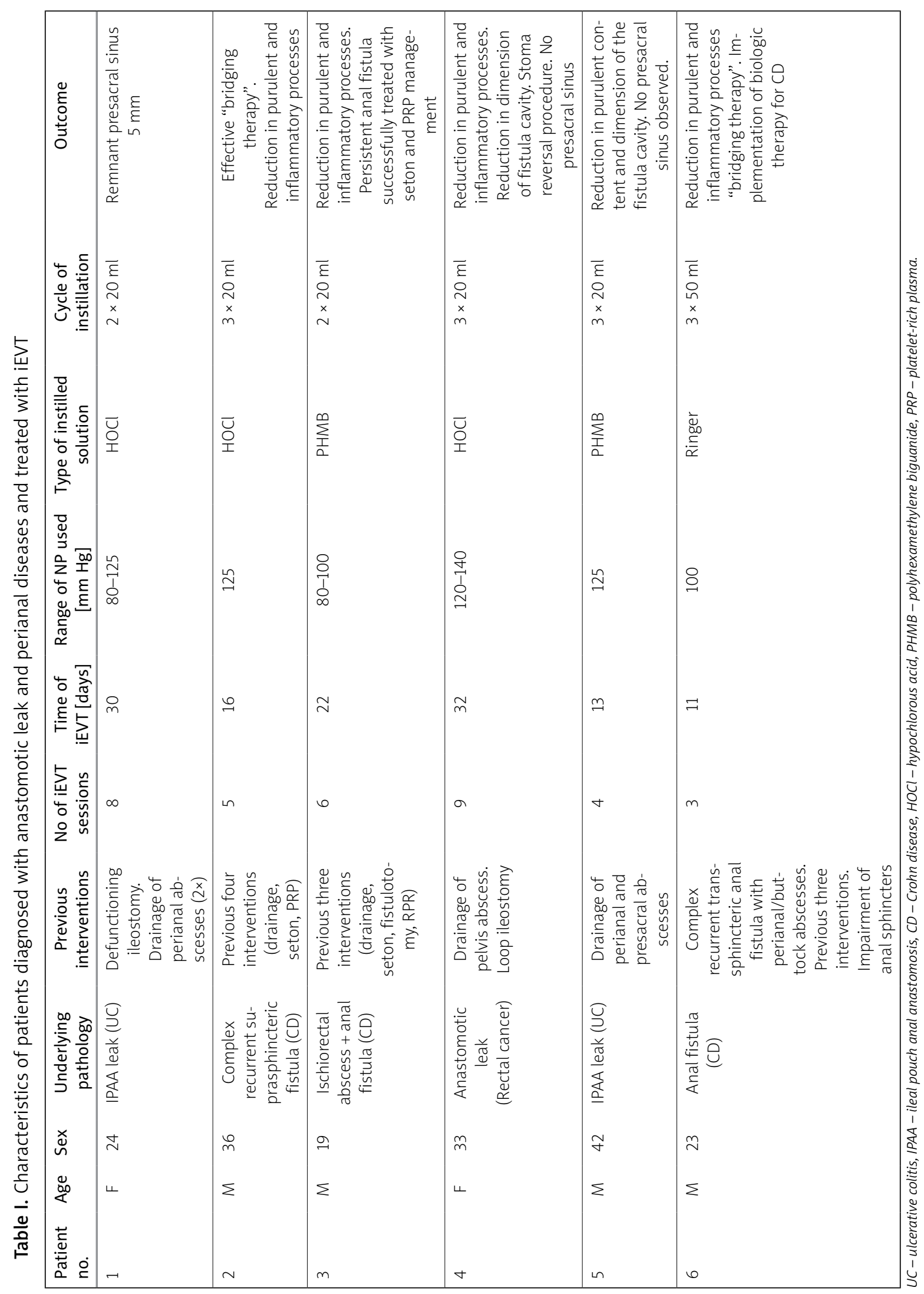



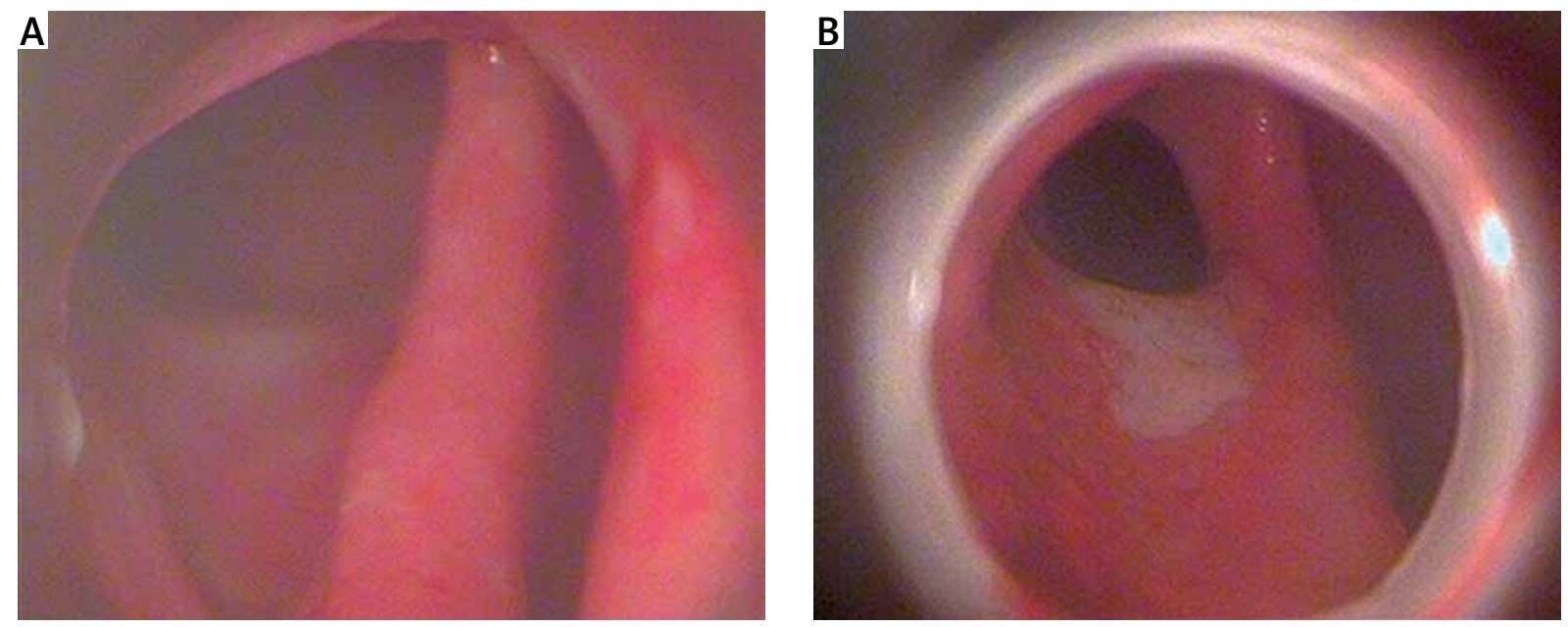

Photo 4. Endoscopic image of fistula cavity: before iEVT management (A) and after seventh session of iEVT (B)

cause of iEVT intervention, diameter of the fistula/ abscess cavity and the exact localization (the risk of unsealed system).

In all patients except one, an antimicrobial solution was used, either $\mathrm{HOCl}$ or PHMB. The appropriate volume of instilled solution was adjust to the preoperative evaluation of fistula/abscess cavity. In some cases we observed leakage of the instilled solution resulting in the need of dressing changes and reduction in volume of the instilled solution. The mean total time of iEVT preparation and application was $44.4 \pm 27.3 \mathrm{~min}$.

In all patients a reduction in purulent content and dimension of the fistula cavity were obtained. Based on endoscopic evaluation, a contraction and revascularization of affected area were revealed (Photo 4). Improvement of general condition characterized by a lack of pelvic pain and fever was achieved. A reduction in C-reactive protein (CRP) and white blood cells (WBC) levels was observed. We did not observe any complications of iEVT. Improvement of general condition and healed fistula cavity allow for restoration of the Gl tract (stoma reversal procedure). In some cases, iEVT was used as a bridge therapy to reduce the purulent and inflammatory process, which allowed for introduction of targeted therapy (e.g. biologics in Crohn disease).

\section{Discussion}

IEVT is a combination of standard endoscopic vacuum therapy and NPWT with instillation (iNPWT). iNPWT creates an optimal environment for wound bed healing. The most important mechanisms of action include flushing and cleansing of the wound bed from microorganisms, cellular debris, cytotoxic molecules and devitalized tissue. Thus, the planktonic bacterial burden decreased, resulting in a reduction of biofilm formation. Moreover, iNPWT up-regulates angiogenesis pathways and maintains a moist wound environment. It was also revealed that iNPWT increased granulation tissue up to $43 \%$ when compared with standard NPWT [8].

Anastomotic leak has a significant impact on patient morbidity and mortality rates. Treatment choices available for anastomotic leak comprise conservative, surgical or endoscopic management. Additionally, the second major surgery is associated with increased morbidity of up to $50 \%$ and risk of having permanent stoma of up to $25 \%$ [9]. Recently, endoscopic management has gained a popularity as a safe therapeutic management. EVT represents a minimally invasive alternative characterized by high success rate of healing of Gl defects without the need of re-operation. The implementation of EVT into the defect cavity allows for effective drainage and minimal risk for developing sepsis. Depending on the dimension of the anastomotic defect, endosponge may be placed directly into the abscess cavity or close to the orifice of the Gl defect covering the leak in case of small anastomotic dehiscence.

Based on our previous experience with NPWT, we found that a combination of the two well-known methods of wound healing (iNPWT, EVT) is a promising strategy for anastomotic leak management. In our opinion, iEVT may be increasingly recognized as a novel, promising technique for enhancing treat- 
ment of $\mathrm{Gl}$ anastomotic insufficiency. It may be applied in various GI defects regardless of the underlying pathology and the type of previous surgery performed.

iEVT may also play an important role in patients with chronic fistula cavities within the Gl tract as a method of treatment or as a bridge therapy before surgical intervention. Potentially, the utility of iEVT therapy allows for local debridement and stimulation of the surrounding tissue, making potential surgical intervention safer and easier in the future.

The presented novel technique is consistent with previous concepts regarding EVT. A self-made EVT was preferably used by some surgeons and endoscopists instead of commercially available EVT due to its lower costs, better flexibility and uniqueness in order to create the optimal EVT for particular individuals. Moreover, recently some authors described many creative amendments and off-label utility of EVT increasing our knowledge and giving strong alternatives to standard EVT and other intraluminal management options [10]. The ongoing improvement in the field of EVT makes this technique a prominent method for anastomotic dehiscence. The described method perfectly meets the strategic direction in endoscopic management of anastomotic leaks. Firstly, it allows us to gain all the well-known advantages of EVT. Secondly, it increases the effectiveness of this method with instilled antiseptic and antimicrobial solutions. Thirdly, for well-experienced endoscopists the expected time to proficiency is relatively low and established approximately after 10 sessions [11]. Moreover, a 2.5 reduction in total cost was recently revealed when compared to the operating room procedures [11].

To our best knowledge, this is the first published report regarding utility of EVT with instillation (iEVT). iEVT is a result of natural evolution of negative pressure wound therapy. Significantly efficient clinical outcomes of NPWT with instillation compared to standard NPWT allow extrapolation of instillation to EVT. Based on the previous studies comparing NPWT and iNPWT and review of evidence and recommendations, iNPWT provides better overall clinical outcomes than standard NPWT. Firstly, iEVT facilitates the loosening, solubilizing and detachment of devitalized tissue, fibrin deposition, wet slough and other tissue and molecules that impair wound healing [12]. In the case of thick exudate, administered antimicrobial solution dissolves the purulent content and facilitates its removal. Secondly, reduction in bacterial bioburden was revealed using iNPWT compared to standard NPWT [13]. Thirdly, the utility of iNPWT significantly influenced increased granulation $(p<0.05)$, greater reduction of wound volume $(p<0.05)$ and higher filling rate of the wound ( $p<$ 0.05) compared to the standard NPWT [14]. Fourthly, iNPWT significantly reduces peri-wound maceration and risk of ingrowth of granulation tissue into the PU foam resulting in wound-bed disruption and mechanical tissue damage during dressing changes [15]. Finally, the length of hospital stay, number of out-patient visits as well as time to final surgical procedure were significantly lower in iNPWT compared to standard NPWT [13].

Some disadvantages could be encountered using iEVT. Firstly, because of the use of instilled solution, this technique should be used with caution in the upper Gl tract due to risk of aspiration if the inappropriate volume of solution is instilled. Secondly, we would like to emphasize that iEVT does not preclude invasive surgical procedures. In the case of a patient's deterioration or lack of improvement of fistula healing, iEVT needs to be abandoned and invasive, surgical management should be implemented. Thirdly, an appropriate volume of antimicrobial solution needs to be instilled, which may be difficult in undiagnosed fistula or in cases of an intraluminal approach (in contrast to intra-cavitary).

\section{Conclusions}

iEVT is a very encouraging, novel method of treatment to heal anastomotic leak and complex perianal fistulae. Further investigations and large cohort studies are needed to establish iEVT effectiveness as a technique for endoscopic salvage therapy. Based on the presented case series, iEVT may play an important role in the management of anastomotic complications and perianal fistulae.

\section{Conflict of interest}

The authors declare no conflict of interest.

\section{References}

1. Blume PA, Walters J, Payne W, et al. Comparison of negative pressure wound therapy using vacuum-assisted closure with advanced moist wound therapy in the treatment of diabetic foot ulcers: a multicenter randomized controlled trial. Diabetes Care 2008; 31: 631-6. 
2. Moues CM, Vos MC, Van Den Bemd GJ, et al. Bacterial load in relation to vacuum-assisted closure wound therapy: a prospective randomized trial. Wound Repair Regen 2004; 12: 11-7.

3. Fleischmann W, Russ M, Westhauser A, Stampehl M. Vacuum sealing as carrier system for controlled local drug administration in wound infection. Unfallchirurg 1998; 101: 649-54.

4. Goss SG, Schwartz JA, Facchin F, et al. Negative pressure wound therapy with instillation (NPWTi) better reduces post-debridement bioburden in chronically infected lower extremity wounds than NPWT alone. J Am Coll Clin Wound Spec 2014; 4: 74-80.

5. Soeters PB, de Zoete JP, Dejong CH, et al. Colorectal surgery and anastomotic leakage. Dig Surg 2008; 19: 150-5.

6. Weidenhagen R, Gruetzner KU, Wiecken T, et al. Endoscopic vacuum-assisted closure of anastomotic leakage following anterior resection of the rectum: a new method. Surg Endosc 2008; 22: 1818-25.

7. Kuehn F, Janisch F, Schwandner F, et al. Endoscopic vacuum therapy in colorectal surgery. J Gastrointest Surg 2016; 20: 328-34.

8. Lessing MC, James RB, Ingram SC. Comparison of the effects of different negative pressure wound therapy modes-continuous, noncontinuous, and with instillation-on porcine excisional wounds. ePlasty 2013; 13: e51.

9. Khan AA, Wheeler JM, Cunningham C, et al. The management and outcome of anastomotic leaks in colorectal surgery. Colorectal Dis 2008; 10: 587-92.

10. Wallstabe I, Nguyen P, Schiefke I, Weimann A. Endoscopic vacuum therapy with open-pore film drainage for colonic anastomotic leakage in a morbidly obese patient. Endoscopy 2019; 51: E51-2.

11. Ward MA, Hassan T, Burdick JS, Leeds SG. Endoscopic vacuum assisted wound closure (EVAC) device to treat esophageal and gastric leaks: assessing time to proficiency and cost. Surg Endosc 2019; 33: 3970-5.

12. Téot L, Florian Boissiere F, Fluieraru S. Novel foam dressing using negative pressure wound therapy with instillation to remove thick exudate. Int Wound J 2017; 14: 842-8.

13. Kim PJ, Attinger CE, Steinberg JS, et al. The impact of negative-pressure wound therapy with instillation compared with standard negative-pressure wound therapy: a retrospective, historical, cohort, controlled study. Plast Reconstr Surg 2014; 133: 709-16.

14. Lessing M, James R, Ingram S. Comparison of the effects of different negative pressure wound therapy modes - continuous, non-continuous, and with instillation - on porcine excisional wounds. Eplasty 2013; 13: e51.

15. Malmsjö M, Ingemansson R. Green foam, black foam or gauze for NWPT: effects on granulation tissue formation. I Wound Care 2011; 20: 294-9.

Received: 29.07.2019, accepted: 11.01.2020. 